\title{
SEGURANÇA DO PACIENTE NA VISÃO DE ENFERMEIROS: UMA QUESTÃO MULTIPROFISSIONAL
}

Marcos Antônio Nunes de Araújo', Wilson Danilo Lunardi Filho², Rosemary Silva da Silveira², José Carlos Souza ${ }^{3}$, Edsom Luis Devos Barlem², Nanci da Silva Teixeira ${ }^{4}$

Objetivo: identificar como o enfermeiro percebe a segurança do paciente na instituição de saúde em que atua. Metodologia: estudo descritivo, de corte transversal, realizado entre janeiro e fevereiro de 2016, em Dourados/MS, com 52,58\% da população de 310 enfermeiros hospitalares. Aplicou-se questionário sociodemográfico com questões vinculadas à segurança do paciente, cujos dados foram submetidos à análise descritiva. Resultados: parte dos enfermeiros apontou menor segurança quanto aos procedimentos, cuidados e administração de medicamentos. Conclusão: compete à equipe multiprofissional atuar na promoção da segurança do paciente, sendo o enfermeiro o profissional apto a identificar e comunicar riscos iminentes, prevenindo a ocorrência de danos e promovendo saúde na sua integralidade, devido à assistência de enfermagem ocorrer ao longo das 24 horas do dia.

Descritores: Segurança do paciente, Gerenciamento de risco, Papel do enfermeiro, Raciocínio clínico.

\section{PATIENT SAFETY IN THE PERPECTIVE OF NURSES: A MULTI PROFESSIONAL ISSUE}

Objective: to highlight how the institutional safety is in the perspective of nurses. Methodology: a cross-sectional descriptive study conducted between January and February 2016, in Dourados City, State of Mato Grosso do Sul, Brazil, with $52.58 \%$ of 310 hospital nurses. A sociodemographic questionnaire with questions about "patient safety" was used. A descriptive and statistical analysis was performed. Results: some of the nurses indicated less safety regarding procedures, care and administration of medication. Conclusion: It is the role of multi professional team to promote patient safety. Nurses are capable of identifying and communicating imminent risks, due to their 24 hours assistance, preventing injuries and promoting health in its entirety.

Descriptors: Patient Safety, Risks Management, Nurse's Role, Clinical reasoning.

\section{SEGURIDAD DE LOS PACIENTES EN LA PERCEPCIÓN DE ENFERMEROS: UNA CUESTIÓN MULTIPROFESIONAL}

Objetivo: identificar cómo la enfermera percibe la seguridad del paciente en la institución de salud en que actúa. Metodologia: estudio descriptivo de corte trasversal hecho de enero a febrero de 2016, en Dourados/MS, con 52,58\% de la población de enfermeros hospitalarias. Se utilizó encuesta sociodemografica respecto la "seguridad del paciente" Se hizo el análisis descriptivo y estadístico. Resultados: parte considerable de los enfermeros indicó menor seguridad cuanto a procedimientos, cuidados y administración medicamentosa. Conclusión: el equipo multiprofesional debe promover la seguridad del paciente, considerando el enfermero como el más apto a identificar y comunicar riesgos inminentes en las $24 \mathrm{~h}$ de su asistencia, evitando danos e promocionando salud en su integralidad.

Descriptores: Seguridad del paciente, Administración de riesgos, Función de los enfermeros, Raciocinio clínico.

${ }^{1}$ Enfermeiro. Doutor. Universidade Estadual do Mato Grosso do Sul. E-mail: marcosojuaralO@gmail.com

${ }^{2}$ Enfermeiro. Doutor. Universidade Federal do Rio Grande.

${ }_{3}^{3}$ Médico. Doutor. Universidade Estadual do Mato Grosso do Sul.

${ }^{4}$ Enfermeira. Mestre. Universidade de Caxias do Sul. 


\section{INTRODUÇÃO}

Cada vez mais, as instituições de saúde precisam ter como meta prioritária atender integralmente e de maneira segura as necessidades de saúde de seus pacientes, sendo muito importante que as atividades desenvolvidas por seus profissionais estejam fundamentadas em pressupostos filosóficos, científicos e metodológicos claros e apropriados para garantir um modelo assistencial de qualidade. A qualidade tem sido uma meta, considerando os constantes aperfeiçoamentos das práticas que buscam a integralidade do cuidado para a satisfação das necessidades de saúde e de segurança de quem depende desses trabalhadores e de seus serviços ${ }^{1}$.

Entretanto, cuidar integralmente, reunindo o conhecimento reflexivo e crítico, exige do enfermeiro raciocinar clinicamente para diagnosticar necessidades de saúde e planejar cuidados de enfermagem de qualidade e seguros. A qualidade do cuidado de enfermagem precisa ser continuamente avaliada, tanto para aprimorar a assistência, como para entender e refletir sobre a segurança do cuidado ofertado ${ }^{2}$. A gerência dos cuidados por parte do enfermeiro produz resultados, implicando diretamente na qualidade e na excelência oferecidas aos pacientes assistidos que, por sua vez, vivenciam a organização do sistema assistencial múltiplo formado pelo quadro de profissionais de enfermagem e saúde. O enfermeiro age na gestão e efetivação do cuidado, na coordenação de recursos humanos e gerência de recursos materiais, no comando e planejamento da assistência e habilitação da equipe de enfermagem, além da avaliação do conjunto das ações ${ }^{3}$.

Em decorrência dos avanços tecnológicos, o hospital tornou-secadavez mais umambienteinseguro e o despreparo de profissionais de saúde pode induzir ao erro na prestação da assistência, causando danos de curto, médio ou longo prazos. Além disso, hospitais lotados, insuficiência de leitos e de profissionais, dupla jornada de trabalho, insuficiência de recursos materiais, dentre outros, aumentam o risco de eventos adversos, comprometendo a segurança do paciente e, em alguns casos, provocando até sua morte. Portanto, o enfermeiro precisa se instrumentalizar com indicadores que avaliem tanto a estrutura quanto o processo e os efeitos de suas ações, procurando estabelecer um olhar mais amplo para a qualidade e segurança da assistência prestada ${ }^{4}$.

Atualmente, na área da saúde, há uma imensa busca pela qualidade e pela segurança, apoiada por políticas públicas, gerando mudanças significativas no Sistema Único de Saúde (SUS) e nos modelos de gestão das instituições ${ }^{5}$. Por sua vez, as modificações demográficas, sociais e econômicas que a sociedade brasileira vivencia, atualmente, têm impactado as categorias de vida e saúde da população e, consequentemente, contribuem com novas ações para o sistema e serviços de saúde do país, influenciando a adaptação a novos perfis de necessidades ${ }^{4}$.

Cabe ressaltar que, embora ainda não tenha o devido reconhecimento como parte importante de um campo autônomo de saber e de intervenção, até mesmo por parcela significativa de seus profissionais, a enfermagem é uma área do saber útil à sociedade, sobretudo, devido ao desenvolvimento de um conjunto de práticas essenciais à vida ${ }^{6}$. Seguindo nessa linha de raciocínio, a realização deste estudo encontrou sua justificativa na importância da temática segurança do paciente, que se mostra recorrente em nosso meio de trabalho. Desse modo, teve-se como objetivo identificar como o enfermeiro percebe a segurança do paciente na instituição de saúde em que atua.

\section{METODOLOGIA}

Trata-se de estudo descritivo, de corte transversal, realizado de janeiro a fevereiro de 2016, no município de Dourados/MS, referência em saúde para 36 municípios da região Sul do estado Mato Grosso do Sul. Considerouse a população de 310 enfermeiros distribuídos entre sete hospitais (dois filantrópicos, dois públicos e três particulares), cuja amostra foi constituída a partir dos critérios de inclusão: trabalhar há, pelo menos, três meses em um ou mais hospitais e não estar afastado por qualquer motivo, no período da coleta das informações.

A amostra foi constituída por 163 enfermeiros (52,58\% do total). Aplicou-se um questionário sociodemográfico que também contemplou questões especificamente vinculadas ao tema da "segurança do paciente" do ponto de vista dos enfermeiros, levando em consideração médicos, fisioterapeutas e enfermeiros como os profissionais diretamente vinculados aos cuidados prestados aos pacientes internados nas unidades dos diferentes hospitais. As questões que constaram no corpo do questionário sociodemográfico estão especificadas no quadro apresentado a seguir:

Quadro - Recorte do questionário sociodemográfico com enfoque na percepção do enfermeiro quanto à segurança do paciente.

\begin{tabular}{|c|c|c|}
\hline QUESTÕES & ALTERNATIVAS POSSÍVEIS & \\
\hline $\begin{array}{l}\text { Você se sente } \\
\text { seguro com } \\
\text { sua assistência } \\
\text { prestada ao seu } \\
\text { paciente? }\end{array}$ & ( ) Sim & ( )Não \\
\hline $\begin{array}{l}\text { Em que área } \\
\text { você observa } \\
\text { que o paciente } \\
\text { está menos } \\
\text { seguro? }\end{array}$ & $\begin{array}{l}\text { ( ) Procedimento médico } \\
\text { ( ) Procedimento de } \\
\text { enfermagem } \\
\text { ( ) Procedimento de } \\
\text { fisioterapia } \\
\text { ( ) Cuidados médicos } \\
\text { ( ) Cuidados de enfermagem } \\
\text { ( ) Cuidados de fisioterapia }\end{array}$ & $\begin{array}{l}\text { ( ) Prescrição } \\
\text { ( ) Prescrição de Fisioterapia } \\
\text { médica } \\
\text { ( ) Administração de } \\
\text { medicamentos pela enfermagem } \\
\text { ( ) Orientação médica } \\
\text { ( ) Orientação de enfermagem } \\
\text { ( ) Orientação de fisioterapia }\end{array}$ \\
\hline
\end{tabular}


Os dados do questionário sociodemográfico foram armazenados em formato Excel, procedendo-se sua análise descritiva.

Oprojeto depesquisafoiaprovadopelosórgãospertinentes CEPAS/FURG n인.2015 e CAAE nㅇ50643215.7.0000.5324.

Realizou-se a coleta de dados somente após a assinatura de Termo de Consentimento Livre Esclarecido (TCLE) pelos participantes e preservou-se o anonimato tanto das pessoas, quanto das instituições envolvidas.

\section{RESULTADOS}

Considerando a variável paciente menos seguro na realização de procedimentos, prescrições, cuidados e orientações obtiveram-se os resultados dispostos na Tabela para Segurança do paciente com relação ao profissional de saúde envolvido, segundo a visão de enfermeiros.

Tabela - Respostas dos enfermeiros sobre "segurança do paciente" na realização de procedimentos, prescrições, cuidados e orientações. Dados da pesquisa, 2016.

\begin{tabular}{|llll|}
\hline \multicolumn{2}{|l|}{$\begin{array}{l}\text { Sente-se seguro com a } \\
\text { assistência que presta } \\
\text { ao seu paciente? }\end{array}$} & $\begin{array}{l}\text { Sim } \\
94,4 \%\end{array}$ & $\begin{array}{l}\text { Não } \\
5,6 \%\end{array}$ \\
\hline \multicolumn{2}{|l|}{ Em que área observa que o paciente está menos seguro quanto a/aos: } \\
\hline Procedimentos & $61,7 \%$ & de enfermagem & de fisioterapia \\
Prescrições & $50,7 \%$ & $8,6 \%$ & $29,6 \%$ \\
Cuidados & $38,18 \%$ & $48,1 \%$ & $1,2 \%$ \\
Orientações & $41,22 \%$ & $27,87 \%$ & $33,93 \%$ \\
\hline
\end{tabular}

\section{DISCUSSÃO}

A enfermagem é uma profissão, cujo objetivo principal é cuidar da saúde e bem-estar do indivíduo, família e comunidade, agindo na promoção, proteção, recuperação e reabilitação da saúde, respeitando princípios éticos e legais no cuidar do ser humano ${ }^{7}$. Possuir conhecimento sobre o corpo e seu perfeito funcionamento é importante para a prevenção e possivel controle dos desequilibrios orgânicos que afetam a saúde. Na visão dos enfermeiros, fica clara a importância atribuída aos fisioterapeutas e médicos, colegas profissionais na atuação no setor de saúde.

A fisioterapia é entendida como aliada na recuperação clínica de pacientes, no âmbito hospitalar, promovendo atividades que buscam a restauração da perda funcional, redução de incapacidades, aprimoramento da funcionalidade, além do tratamento e prevenção de complicações respiratórias, mediante massagem, exercícios e uso de agentes eletrofísicos. Há o entendimento de que ao profissional médico, por sua vez, compete realizar consultas e atendimentos, procedimentos técnicos, tratar e implementar ações para recuperação, manutenção e promoção da saúde, coordenar programas e serviços de saúde, efetuar perícias, auditorias e sindicâncias, elaborar documentos e difundir conhecimentos da área médica e da saúde. Assim, conforme evidenciado na visão dos enfermeiros, as três profissões e os respectivos cursos de graduação, apesar de estarem na mesma área e atuarem diariamente com os pacientes, são diferentes nos objetivos de suas formações, atribuições e ações.

Sobre as evidências de elementos necessários para a promoção da cultura de segurança institucional, identificouse que os enfermeiros reconhecem os principais riscos (físicos, químicos, assistenciais, clínicos e institucionais) aos quais os pacientes sob seus cuidados estão expostos. Os riscos apontados são inquietantes, pois evidenciam a qualidade da assistência. Entretanto, após o seu levantamento, precisam ser analisados para elucidar as possiveis causas, direcionando reflexões e possiveis propostas de solução e de educação permanente às equipes de enfermagem e de saúde no serviço ${ }^{8}$. A atitude de compartilhar a identificação de riscos pode ser considerada uma primeira e importante estratégia para o estabelecimento de uma cultura de segurança nas instituições de saúde.

O fisioterapeuta tem como principal instrumento de trabalho o seu próprio corpo. Geralmente, a sua atividade é dinâmica, com grande deslocamento do centro de gravidade, o que the exige bom equilibrio para atuar nas diversas posturas. Além disso, faz uso de agentes como água e calor, instrumentos e equipamentos mecânicos e elétricos no auxílio terapêutico baseado na cinesioterapia, massoterapia, manipulação, eletroterapia, hidroterapia e mecanoterapia. Portanto, em seu trabalho realiza procedimentos e apresenta situações que podem contribuir potencialmente para a diminuição da segurança do paciente?.

A análise estatística sobre a segurança do paciente associada às informações sociodemográficas levantadas indicam que os enfermeiros concursados e com mais de cinco anos de experiência percebem o paciente menos seguro, quando os cuidados são realizados por médicos, o que pode advir da maturidade profissional para essa observação. Em pesquisa sobre a segurança do paciente na terapêutica medicamentosa e a influência da prescrição médica nos erros de dose, menciona-se que, quando o prescritor não especifica a dosagem requerida em miligramas, utilizandose de abreviaturas ou siglas nas receitas, aumenta o risco de o paciente receber uma dose medicamentosa diferente da necessária, uma vez que fármacos como a dipirona, por exemplo, apresentam várias formas farmacêuticas no mercado (ampolas de $1 \mathrm{ml} / 500 \mathrm{mg}$, de $2 \mathrm{ml} / 500 \mathrm{mg}$ e de $5 \mathrm{ml} / 500 \mathrm{mg})$. Além disso, a identificação adequada do paciente também se faz necessária, uma vez que pessoas com nomes parecidos, internados em uma mesma enfermaria e 
recebendo um mesmo medicamento, em doses diferentes, ou tendo necessidade de determinados cuidados, podem ser confundidos, prejudicando os respectivos tratamentos ${ }^{10}$.

Outro estudo enfatiza que a qualidade do cuidado de enfermagem reflete a qualidade e a segurança da assistência ao paciente, sendo que métodos de pesquisa como a pesquisa fotográfica, por exemplo, podem auxiliar no gerenciamento de riscos no trabalho com medicações, ajudando a abreviar o tempo de internações hospitalares, diminuindo a incidência de incapacitações temporárias ou permanentes e prevenindo mortes desnecessárias ${ }^{11}$. Destaca-se, ainda, estudo que aponta ser a enfermagem uma profissão ímpar, em função da oferta e prestação de seus serviços, nas 24 horas do dia ao paciente que se encontra debilitado em seu leito, aguardando uma assistência segura ${ }^{12}$.

Na área de saúde, há interesse crescente pela qualidade em todos os setores. Usuários mais bem informados tornam-se mais exigentes, gestores buscam otimizar o uso dos recursos humanos e materiais e os profissionais de saúde, além de quererem sobreviver profissionalmente no mercado, preocupam-se com a segurança e qualidade do trabalho que realizam, devido ao compromisso ético intrínseco a essa área e que envolve cuidar do bem mais valioso das pessoas que é sua saúde ${ }^{13}$

A avaliação da qualidade de

$$
\begin{aligned}
& \text { "Em pesquisa realizada } \\
& \text { em } 2014, \text { foi identificado } \\
& \text { que em nenhum dos } \\
& \text { cuidados de enfermagem } \\
& \text { foi alcançado um nivel } \\
& \text { desejável de assistência } \\
& \text { de enfermagem" }
\end{aligned}
$$

deenfermagem possuem dificuldade emampliaraabrangência de seus cuidados para incluir aqueles que atendam com maior propriedade outras necessidades psicobiológicas, além das necessidades psicossociais e psicoespirituais dos pacientes ${ }^{15}$.

Com o objetivo de contribuir com os avanços científicotecnológicos acelerados, ampliando 0 atendimento às exigências dos clientes da saúde, em meio à competitividade no mundo da globalização, as instituições de saúde, a exemplo das demais prestadoras de serviços, vêm buscando assegurar a qualidade do trabalho que realizam, por meio, dentre outros, de ações sistemáticas e periódicas de avaliação ${ }^{16}$

Por sua vez, as orientações de saúde são consideradas importantes para os pacientes em tratamento e que estão em busca da melhoria da qualidade de saúde. Nesse processo, tornam-se uma tecnologia importante alicerçada no pensamento reflexivo crítico dos profissionais de saúde, por meio do diálogo e do conhecimento socializado ${ }^{15}$.

Além das orientações,
os cuidados prestados
antes, durante e depois de
internações e tratamentos
mostram-se relevantes para
o alcance e a manutenção da
segurança dos pacientes ${ }^{17}$.
Entretanto, como as pesquisas
têm apontado, muito pouco
ou quase nada adianta o
paciente ser cuidado por uma
equipe multiprofissional, que
visa à promoção da sua saúde
e do seu bem-estar, se a
estrutura não colabora com o
funcionamento adequado para
a prestação de uma assistência
na área da saúde, em todo

o mundo, impulsionada pela demanda de financiadores, prestadores de serviços, profissionais e o público em geral. Estudos sobre a qualidade do cuidado hospitalar vêm ganhando volume, no Brasil, tendo destaque aqueles que exploram a abordagem do processo e do resultado do cuidado. Evidenciou-se que, nos estudos desenvolvidos no país, a preocupação com a abordagem dos recursos da estrutura assistencial prevalece justificada pela deficiência na oferta de profissionais e ausência de ambientes adequados à prestação dos cuidados ${ }^{14}$.

Em pesquisa realizada em 2014, foi identificado que em nenhum dos cuidados de enfermagem foi alcançado um nivel desejável de assistência de enfermagem e que, apenas, dois grupos de cuidados foram considerados seguros higiene e conforto físico e nutrição e hidratação - ambos pertencentes à categoria das necessidades humanas básicas psicobiológicas. Essa conclusão mostra que os trabalhadores de qualidade e segura ${ }^{12}$

Considerando as limitações deste estudo, que dizem respeito ao curto período de coleta de dados (janeiro e fevereiro de 2016) e à circunscrição da população tida como foco (enfermeiros da rede hospitalar de Dourados/MS), entende-se que as visões e perspectivas manifestadas pelos profissionais necessitam ser validadas, por meio de novos empreendimentos que delineiem os pontos de vista de outras populações de enfermeiros sobre a sua prática e de seus colegas de outras profissões no ambiente de trabalho que compartilham.

\section{CONCLUSÃO}

A enfermagem é uma profissão que busca a promoção do do distúrbio de saúde, podem deparar-se com internações e outros procedimentos que podem contemplar cuidados bem-estar de seus clientes, os quais, ao longo do percurso 
intensos e ininterruptos, com todo um aparato tecnológico para monitoramento ou realização de investigações, visando a outros diagnósticos. Por esse motivo, compete não somente ao enfermeiro, mas a toda equipe multiprofissional atuar na promoção da segurança desses pacientes mais expostos a riscos e propensos a sofrer algum dano. Nesse sentido, tais afirmações são corroboradas pelos achados desta pesquisa, demonstrando a visão dos enfermeiros sobre os riscos iminentes ao tratamento em saúde e a importância do diálogo e da construção de vias para a ação multidisciplinar que promovam medidas para a garantia da segurança e do bem-estar dos pacientes.

Desse modo, a segurança do paciente, em todas as suas dimensões, vem se tornando uma preocupação constante para o setor da saúde, motivo pelo qual é um tema que precisa ser intensamente debatido na atualidade, devido às evidências do impacto da ocorrência dos erros e dos eventos adversos nos sistemas de saúde. Assim, percebese que a segurança do paciente não é uma problemática individual, tampouco de uma única categoria profissional, mas fruto de um processo que envolve a necessidade da transformação institucional. Promover debates sobre a segurança do paciente e instituir cuidados seguros e que não causem danos evitáveis mostram-se mais do que necessários, no sentido de estabelecer estratégias, com vistas a proporcionar uma assistência de qualidade e implantar uma cultura de segurança nas instituições de saúde.

\section{REFERÊNCIAS}

1. Pertence PP, Melleiro MM. A implantação de uma ferramenta de gestão da qualidade em um hospital universitário. Rev. Esc. Enferm. da USP, São Paulo, v. 44, n. 4., p. 1024-1031, dez. 2010

2. Vituri DW, Matsuda M. Content validation of quality indicators for nursing care evaluation. Rev. Esc. Enferm. da USP. São Paulo, v. 43, n. 2.. p. 429-437. jun. 2009.

3. Santos BP, Ferreira GB, Soares MC, Meincke SMK, Könzgen SM. Ensino de enfermagem no Brasil: do advento do sistema Nightingale ao cenário científico. Hist. Enf. Rev. Eletr. 2014 Aug.-Dec. [cited 2017 Jan 22]: 5(2):31022. Available from: http://pesquisa.bvs.br/brasil/resource/pt/bde-26779.

4. Vieira FS, Caldana RHL, Corradi-Webster CM. Relatos de auxiliares de enfermagem psiquiátricos sobre suas práticas e sobre pessoas internadas por uso compulsivo de substâncias psicoativas. Saúde transform. soc. 2013 [cited 2017 Jan 27]; (4):139-48. Available from: http://incubadora.periodicos. ufsc.br/index.php/saude etransformacao/article/view/2244

5. Padilha EF, Matsuda LM. Qualidade dos cuidados de enfermagem em terapia intensiva: avaliação por meio de auditoria operacional. Rev. bras. Enferm., Brasilia, v. 64, n. 4, p. 684-691, ago. 2011.

6. Amendoeira J. Ensino de enfermagem: perspectivas de desenvolvimento. PensarEnferm., [s.l.], v. 13, n. 1, p. 2-13, 1 sem. 2009.

7. Pizzoli LML. Qualidade de vida no trabalho: um estudo de caso das enfermeiras do Hospital de Heliópolis. Ciênc. Saúde Colet. 2005 [citado em 19 dez 2016]; 11(4):1065-2. DOI: http://dx.doi.org/10.1590/S141381232005000400028

8. Oliveira RM, Leitão IMTA, Silva LMS, Figueiredo SV, Sampaio RL, Gondim MM. Strategies for promoting patient safety: from the identification of the risks to the evidence-based practices. Esc. Anna Nery. 2014 [cited 2016 Nov 10]; 18(1):122-9. Available from: http://www.scielo.br/pdf/ean/v18nl/ en_1414-8145-ean-18-01-0122.pdf.

9. Cárdia MCGA. Organização dos Serviços de Fisioterapia no Setor Público de João Pessoa/PB [trabalho de conclusão de curso] 1996. Universidade Federal da Paraíba, Especialização em Saúde do Trabalhador, João Pessoa, PB. Brasil.
10. Gimenes FRE, Mota MLS, Teixeira TCA, Silva AEBC, Optotz SP, Cassiani SHB. Segurança do paciente na terapêutica medicamentosa e a influência da prescrição médica nos erros de dose. Rev Latino-Am Enferm. 2010 Nov.Dec. [cited 2016 Oct 27]; 18(6): 07 telas. Available from: http://www.scielo. br/pdf/rlae/v18n6/pt_03.pdf.

11. Raduenz AC, Hoffmann PP, Raduenz V, Sasso GTMD, Maliska ICA, Marck PB. Cuidados de enfermagem e segurança do paciente: visualizando a organização, acondicionamento e distribuição de medicamentos com método de pesquisa fotográfica. Rev. Latino-Am. Enferm. 2010 Nov.-Dec. [cited 2016 Oct 30]: 18(6):10 telas. Available from: http://www.scielo.br/pdf/ rlae/v18n6/pt_02.

12. Costa DVS, Fragoso LVC, Queiroz PA, Carvalho SMA, Costa DVS, Freitas MMC. Nursing Contributions to the Patient's Safery in The Intensive Care Unit: An Intregrave Review. Rev enferm UFPE. 2016 Jun. [cited 2017 Jan 13]; 10(6):2177-88. Available from: http://www.revista.ufpe.br/ revistaenfermagem/index.php/revista/article/ viewArticle/7051.

13. Agência Nacional de Vigilância Sanitária (BR). Assistência Segura: Uma Reflexão Teórica Aplicada a Prática. Brasilia (BR): ANVISA; 2013 [cited 2016 Mar 17]. Available from: http://www20.anvisa.gov.br/segurancadopaciente/ images/documentos/livros/Livrol-Assistencia_Segura.pdf.

14. Machado JP, Martins ACM, Martins MS. Avaliação da qualidade do cuidado hospitalar no Brasil: uma revisão sistemática. Cad. Saúde Pública. 2013 Jun. [cited 2016 Nov 17]; 29(6): 1063-82. Available from: http://www. scielo.br/pdf/csp/v29n6/a04v29n6.pdf.

15. Freitas JS, Silva AEC, Minamisava R, Bezerra ALQ, Souza MRG. Qualidade dos cuidados de enfermagem e satisfação do paciente atendido em um hospital de ensino. Rev. Latino-Am. Enferm. 2014 May.-Jun. [cited 2016 Dec 17]: 22(3):454-60. DOI: http://dx.doi.org/10.1590/0104-1169.3241.2437.

16. Pena MM, Silva EMS, Tronchin DMR, Melleiro MM. The use of the quality model of Parasuraman, Zeithaml and Berry in health services. Rev. esc. Enferm. USP. 2013 [cited 2017 Feb 04]; 47(5):1227-32. DOI: http://dx.doi. org/10.1590/s0080-62342013000 0500030 .

17. Chistoforo BEB, Carvalho DSS. Cuidados de enfermagem realizada ao paciente cirúrgico no período pré-operatório. Rev Enfermagem USP. 2009 [cited 2016 Nov 2016]; 43(1):14-22. Available from: http://www.scielo.br/pdf/ reeusp/v43n1/02.pdf 